New York, NY and other centers in Germany, Belgium and the U.S. Symptoms manifested within or soon after the first year of life and muscle biopsies showed ragged red fibers and decreased respiratory chain activity. There was good correlation between clinical severity and degree of mtDNA depletion in muscle. An infant who died at 2 months had lactic acidosis and less than $2 \%$ of the normal level of mtDNA, while 4 children with relatively milder myopathy had no lactic acidosis and 8-34\% residual mtDNA (Tritschler H-J et al. Mitochondrial myopathy of childhood associated with depletion of mitochondrial DNA. Neurology Jan 1992; 42:209-217). (Reprints: Dr. Eric A. Schon, Department of Neurology, Rm. BB324, Columbia University, 630 West 168 th St., New York, NY 10032.)

COMMENT. Depletion of mtDNA is considered a distinct entity distinguished from other known mitochondrial disorders with COX deficiency or with multiple respiratory chain defects (fatal and benign infantile myopathies, Leigh's syndrome, Kearns-Sayre syndrome). The principal clinical features of patients with mtDNA depletion and mitochondrial myopathy are weakness, hypotonia and respiratory distress.

\title{
MITOCHONDRIAL MYOPATHY WITH DNA DELETIONS
}

Deletions of mitochondrial DNA (mtDNA) are reported in 19 of 56 patients with mitochondrial myopathy examined in the Department of Neurology and Neuromuscular Research Laboratory, Mayo Clinic, Rochester, MN. All 19 patients had progressive external ophthalmoplegia and 12 had complete or partial Kearns-Sayre syndrome. The age at onset varied from 4 to 48 years, 10 presenting in childhood. Patients with more than $50 \%$ deleted mtDNA had an earlier onset of symptoms and a higher proportion of ragged red fibers and cytochrome c oxidase negative fibers than patients with less than $50 \%$ deleted mtDNA (Yamamoto $\mathrm{M}$ et al. Mitochondrial DNA deletions in mitochondrial cytopathies: observations in 19 patients. Neurology Nov 1991; 41:1822-1828). (Reprints: Dr. Andrew G. Engel, Department of Neurology, Mayo Clinic, Rochester MN 55905.)

COMMENT. This paper confirms that large scale mtDNA deletions are present in a high proportion of patients with mitochondrial myopathy associated with progressive external ophthalmoplegia and that these deletions are a hallmark of Kearns-Sayre syndrome. Kearns-Sayre syndrome consists of progressive external ophthalmoplegia, pigmentary retinopathy, cardiac conduction abnormalities, and mitochondrial myopathy involving facial, cervical and limb muscles and increased CSF protein.

\section{LEIGH'S SYNDROME WITH TWO MITOCHONDRIAL DEFECTS}

A female infant with a biochemical defect of the respiratory chain and of $\beta$-oxidation and neuropathological changes typical for Leigh's disease is reported from the Department of Neurology, University of Würzburg, Departments of Pediatrics and Pathology, University of Homburg, and Department of Pediatrics, University of Freiburg, Germany. The infant was 
seen at age 2 months because of hypotonia, delayed motor development and lactic acidosis. Decreased activities of both cytochrome c oxidase and long chain acyl coenzyme A dehydrogenase were found in a muscle specimen of 11 months and in a liver specimen obtained post mortem at 13 months. Myoclonic seizures accompanied by multifocal EEG epileptic discharges were treated with valproate. Seizures were controlled, but her general condition deteriorated within 2 months, respiratory insufficiency worsened and the acidosis became intractable. Post mortem examinations showed severe demyelination in the capsula interna, calcification in the brain stem ganglia, and gliosis in the pons and medulla. The liver was enlarged with centrolobular fatty infiltration. A rare occurrence of two independent mitochondrial enzyme defects is suggested (Reichmann H et al. Cytochrome c oxidase deficiency and long-chain acyl coenzyme A dehydrogenase deficiency with Leigh's subacute necrotizing encephalomyelopathy. Ann Neurol Jan 1992; 31:107-109). (Correspondence: Dr. Reichmann, Department of Neurology, University of Würzburg, Josef-Schneider-Strasse 11, D-8700 Würzburg, Germany.)

COMMENT. Valproate administration was not considered the cause but may have worsened the palmitoyl CoA dehydrogenase deficiency. Leigh's syndrome, first described in 1951, appears to be non-specific biochemically as well as clinically. Consistent early clinical features in the infantile cases are a quiet immobility with lack of crying and hypotonia. In addition to the COX deficiency and dehydrogenase deficiency described above, defects of the pyruvate dehydrogenase multi-enzyme complex and pyruvate carboxylase have been reported.

\section{FAMILIAL SPASTIC PARAPARESIS AS A MITOCHONDRIAL DISORDER}

A girl presenting at 6 years with familial spastic paraparesis and having deficiencies of respiratory chain enzyme complex I, III and IV is reported from the Departments of Pediatrics and Neurology, Loyola University Medical Center, Maywood, IL. Her gait was clumsy and her maternal grandmother had a spastic gait with onset at age 40 . Deep tendon reflexes were brisk at the knees. Plantar responses were extensor bilaterally and ankle clonus was unsustained. The tone was increased in both lower extremities. The gait was wide based with hip circumduction. A 3 centimeter cyst in the left parietal region on MRI appeared to be coincidental. Efforts to treat the deficiencies of mitochondrial enzymes with various respiratory chain co-factors (coenzyme $\mathrm{Q}$, vitamin $\mathrm{K}$ and $\mathrm{C}$ ) and a mitochondrial substrate entry carrier (carnitine) were rewarded with no further progression of symptoms and some mild improvement clinically. The mitochondria were structurally normal by electron microscopy and ragged red fibers were absent on muscle biopsy (Beltran RS, Coker SB. Familial spastic paraparesis: a case of a mitochondrial disorder. Pediatr Neurosurg Oct 1991; 16:40-42). (Reprints: Dr. Beltran, Department of Pediatrics, Loyola University Medical Center, 2160 South First Ave., Maywood, IL 60153.)

COMMENT. Familial spastic paraparesis or Stümpell's disease is characterized by a progressive gait disturbance with lower extremity 